\title{
Are technicolor models compatible with a very heavy top quark?
}

\author{
Martin B. Einhorn and Daniel Nash \\ Institute for Theoretical Physics, University of California, Santa Barbara, CA 93106, USA \\ and \\ Randall Laboratory of Physics, University of Michigan, Ann Arbor, MI 48109-1120, USA
}

Received 6 August 1991

Accepted for publication 27 September 1991

\begin{abstract}
We consider the phenomenological constraints on technicolor and extended technicolor (ETC) interactions required to give a realistic quark-lepton spectrum consistent with limits on flavorchanging neutral currents. Treating ETC interactions as higher-dimensional vertices in an effective-ficld thcory of technicolor, we outline a phenomenologically acceptable technicolor model with a walking $\mathrm{SU}_{2}$ technicolor and a single generation of technifermions. Without fine-tuning or violating known constraints, we argue that both the strange-quark mass and a top-quark mass on the order of $100 \mathrm{GeV}$ can be plausibly accommodated However, the precise ETC group and the dynamical mechanism responsible for its breaking are not determined.
\end{abstract}

\section{Introduction}

From a fundamental point of view, one of the least satisfactory aspects of the electroweak theory is its method of accounting for quark and lepton masses and the Cabibho-Kobayashi-Maskawa (CKM) mixing angles. Although no symmetries are imposed other than $\mathrm{SU}_{3}^{\mathrm{C}} \otimes \mathrm{U}_{1}^{\mathrm{Y}}$ gauge invariance, every allowable Yukawa coupling is introduced and given whatever values are necessary to fit the data. This is perfectly acceptable, but, even though there are no known internal or phenomenological contradictions, it is puzzling that the range of masses vary over five orders of magnitude from the mass of the electron to that of the top quark, which, while as yet unknown, must exceed $89 \mathrm{GeV}$ [1]. Indeed, the splitting between the bottom- and top-quark masses suggests that the classification by generations is only remotely related to the mass spectrum. Moreover, the pattern of mixing angles in the CKM matrix is also completely unaccounted for and, in principle, is unrelated to the masses. Results from SLC and LEP imply that there are no more generations of fermions having massless or light neutrinos $\left(m_{\nu} \leqslant M_{Z} / 2\right)$ other than the 


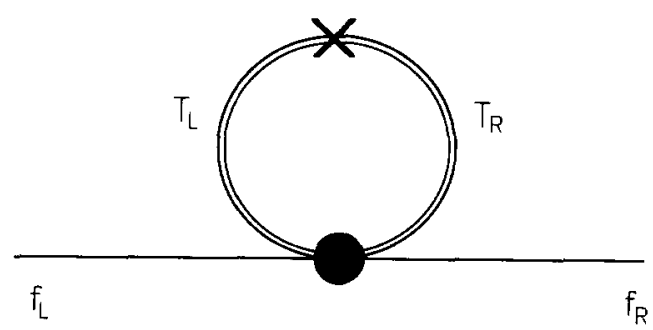

Fig. 1. Fermion mass generated by an ETC interaction. The single lines are fermions, and the double lines are technifermions. The crosses represent technifermion self-energies, and the dot is the four-fermion operator generated by ETC exchange.

three varieties that are already known [2]. Thus, the problem that lies before us is to understand the origin of these fundamental parameters.

Another central question of the Standard Model concerns the mechanism underlying electroweak symmetry breaking embodied in the Higgs sector. One of the most attractive ideas for a dynamical mechanism responsible for the breaking is known as the technicolor (TC) scenario [3], in which a set of technifermions and a new vectorlike gauge interaction mimic the chiral symmetry breaking mechanism of quantum chromodynamics (QCD.) However, this must be supplemented to account for the fermion masses, which have been ascribed to yet another strong interaction commonly termed an extended technicolor (ETC) interaction [4,5]. (See fig. 1.) To be more precise and to introduce notation, the generic form of the quark and lepton masses is

$$
m_{f}=\sum_{F} \lambda_{f F} \frac{\left.\langle\bar{F} F\rangle_{M_{\text {ET }}^{f F}}^{f F}\right)^{2}}{\left(M_{\text {ETC }}^{f F}\right.}
$$

where $\lambda_{f F}$ represents some effective four-fermion coupling constant associated with transitions between the ordinary fermion of flavor $f$ and the technifermion of flavor $F . M_{\text {ETC }}^{f F}$ is the mass of a particle that communicates between the fermions and technifermions, and $\langle\bar{F} F\rangle_{M_{\mathrm{ETC}}}$ is the corresponding technifermion condensate. If $M_{\mathrm{ETC}}^{f F}$ corresponds to an ETC vector boson, then the coupling $\lambda_{f F}$ is of order of the square of the ETC gauge coupling $g_{\mathrm{ETC}}^{2}$. The ratio $v_{\mathrm{ETC}}^{f F} \equiv M_{\mathrm{ETC}}^{f F} / \sqrt{\lambda_{f F}}$ will be referred to as the ETC scale associated with the contribution of the condensate $\langle\bar{F} F\rangle$ to the mass of fermion $f$.

While aesthetically attractive, this scenario has faltered phenomenologically, as will be reviewed in sect. 2 , because it is seemingly incapable of simultaneously generating large enough quark or lepton masses while at the same time adequately suppressing strangeness-changing neutral currents. There are other phenomenological issues as well concerning the technihadron spectrum, and especially, the masses of the collective modes known as pseudo-Goldstone bosons (PGB) arising from chiral symmetry breaking among the technifermions. Indeed, the technicolor 
concept languished until recently because of its apparently irreconcilable phenomenological contradictions. It has been revived by dynamical mechanisms that enhance the value of the technifermion condensate relative to the weak scale. One was the notion of a finite ultraviolet fixed point [6]. A second idea, closer to more familiar dynamics, has been termed "walking technicolor" [7], since it is a consequence of a very slowly running technicolor coupling constant between the scale $\Lambda_{\mathrm{TC}}$ at which the TC coupling is strong and the scale $v_{\mathrm{ETC}}$ associated with ETC interactions. The requirement that the technicolor beta-function be small is a stringent requirement on the TC group $\mathrm{G}^{\mathrm{TC}}$ and technifermion representation. A third mechanism, referred to loosely as "four-fermion" enhancement [8,9], considers the possibility that the effective four-technifermion interactions resulting from ETC interactions are relatively strong. Actually, these last two mechanisms are closely intertwined. Assuming as we must that the TC group is a subgroup of the ETC group, unless one considers very large groups, the only natural way one can imagine such relatively strong ETC interactions "matching" onto the TC interaction below the ETC symmetry breaking scale is if indeed the TC coupling is relatively large at the ETC scale. Thus the dynamics of four-fermion enhancement presupposes a persistent (walking or, at least, rather slowly running) technicolor coupling.

Despite these mechanisms that have breathed new life into dynamical models of electroweak symmetry breaking, no phenomenologically acceptable ETC theory has been produced [10]. Part of the problem may be that the ETC dynamics involves unfamiliar new mechanisms. Whereas the TC theory is supposed to be qualitatively similar to QCD scaled up by a factor of 1000 , the ETC interactions are non-vector-like *, necessitated by having to give different masses to the two members of an $\mathrm{SU}_{2}^{\mathrm{W}}$ doublet. It may be that the mechanism of condensate formation in non-vector-like theories is an unfamiliar one, and this ignorance contributes to our inability to specify the ETC group. Moreover, it is not obvious whether ETC symmetry breaking is to be associated with a single scale or a multitude of scales or whether the ETC symmetry group is a simple group. Regardless of what the underlying dynamics is, below the ETC scales, one will face an effective field theory consisting of fermions (quarks, leptons, techniquarks, technileptons) and TC gauge bosons interacting in the usual gauge invariant manner, plus higher-dimensional terms, in particular, four-fermi interactions, resulting from the decoupling of the heavy ETC vector bosons. In addition, it may be necessary to include pseudo-Goldstone bosons in the effective lagrangian, although we will not discuss their properties here. It is possible and challenging to

\footnotetext{
* This is true regardless of whether the ETC group tumbles via the maximally attractive channel or not. One might imagine that vector-like ETC interactions would be spontaneously rather than explicitly broken, but there is substantial evidence that vector-like gauge symmetries do not undergo spontaneous symmetry breakdown [11].
} 
attempt to characterize the general form that this effective field theory must take to be phenomenologically successful. That is what we will begin to do in this paper. In particular, given the weak scale, we wish to address the question of whether a very large top-quark mass can be accounted for at all in this framework and how the disparate masses, both intra- and intergenerational, of the known fermions could arise naturally in such an effective field theory.

We would like to proceed in as model-independent a fashion as possible, but in fact we can go only a short way before choices become necessary. Without specifying the ultimate ETC group or the precise symmetry-breaking mechanism giving rise to the various ETC scales, we will assume that the generic ETC picture is basically correct, in which ordinary quark and lepton masses are driven by the technifermion condensate responsible for electroweak symmetry breaking (fig. 1.) We assume that ultimately, at some extremely high momentum scale, the theory consists of only fundamental fermion fields interacting via gauge bosons. This ultimate "unified" ETC group must be subject to the constraints described by Eichten and Lane [5]. In particular, the left-handed fermions must lie in a single irreducible representation of the group, while the right-handed fermions lie in at most two irreducible representations. So, quarks, leptons, and technifermions must become unified and transform into each other under some ETC interactions. Among other things, this implies that color $\mathrm{SU}_{3}^{\mathrm{C}}$ and weak $\mathrm{SU}_{2}^{\mathrm{L}}$ interactions eventually must be included in the ETC group. However, unlike Eichten and Lane, we imagine that this unified ETC group is spontaneously broken at many different scales, so that, roughly, the lighter (heavier) fermions are to be associated with the higher (lower) scales. This is as was anticipated in tumbling gauge-theories [12], but we will not concern ourselves here with the precise dynamics responsible for the breaking.

Instead of making a guess at the ultimate ETC group and its pattern of breaking at successively lower energy scales, we will instead attempt to work our way up from the lowest scales. At least up to the lowest scale $M_{\mathrm{ETC}}^{(\mathrm{t})}$ responsible for the top mass, for reasons to be reviewed below, we shall entertain walking technicolor and strong four-fermion interactions. This scale will turn out to be on the order of 5-10 TeV. At this energy, color $\mathrm{SU}_{3}^{\mathrm{C}}$ or the weak $\mathrm{SU}_{2}^{\mathrm{L}}$ cannot form subgroups of the effective ETC symmetry group at this scale, since their couplings are relatively weak. This implies that the ETC gauge bosons at this scale cannot carry color. So it is natural to suppose that the technifermions responsible for quark masses carry color while those responsible for lepton masses do not. The simplest model of this sort has a single generation of technifermions $\mathrm{U}, \mathrm{D}, \mathrm{N}, \mathrm{E}$ whose quantum numbers other than technicolor are identical to those of a single generation of quarks and leptons [13]. Unlike ordinary neutrinos, which may or may not be massive, since we do not want any massless technifermions, we must suppose that there are righthanded technineutrinos.

Below the lowest ETC scale $M_{\mathrm{ETC}}^{(\mathrm{t})}$, the underlying symmetry of the effective 
field theory is $\mathrm{SU}_{3}^{\mathrm{C}} \otimes S \mathrm{SU}_{2}^{\mathrm{L}} \otimes \mathrm{U}_{1}^{\mathrm{Y}} \otimes \mathrm{G}^{\mathrm{TC}}$. As in $\mathrm{QCD}$, the technicolor interactions are assumed to be vector-like, so they will respect an additional global $\mathrm{SU}_{2}^{\mathrm{R}}$ chiral symmetry that is ultimately responsible for the approximate custodial weak isospin $\mathrm{SU}_{2}$ that gives the heralded relation $\rho \equiv M_{\mathrm{W}}^{2} / M_{\mathrm{Z}}^{2} \cos ^{2} \theta \approx 1$. If ETC interactions also respected this custodial symmetry, then the splittings between quarks and leptons would have to be ascribed to color forces *, and the splittings between members of the same $\mathrm{SU}_{2}^{\mathrm{L}}$ doublet would somehow have to come from hypercharge. It is amusing that, in the ladder approximation, the signs of all these effects are in the right direction, but their magnitude is too small to account for observations, even allowing for a certain amount of fine tuning ${ }^{\star}$. Thus, it must be the case that the ETC interactions themselves, even at the scale $M_{\mathrm{ETC}}^{(1)}$, violate the custodial symmetry and are not vector-like.

What scales are to be associated with each quark and lepton? Just as in the Standard Model where the Yukawa coupling for each flavor is arbitrary, so also is the associated ETC scale to be associated with each quark and lepton. However, the disparity in ETC scales associated with two members of a weak doublet, such as the top and bottom quarks, must arise in a definite fashion, since, in this scenario, the unbroken ETC symmetry operative just above $M_{\mathrm{ETC}}^{(\mathrm{t})}$ cannot contain the $\mathrm{SU}_{3}^{\mathrm{C}} \otimes \mathrm{SU}_{2}^{\mathrm{L}} \otimes \mathrm{U}_{1}^{\mathrm{Y}}$ group. We must assume that the $b_{\mathrm{R}}$ field does not participate in four-fermion interactions associated with the $M_{\mathrm{ETC}}^{(\mathrm{t})}$.

The conventional association of a scale with the suppression of flavor-changing neutral currents assumes there are Born-interactions mediating transitions such as $\mathrm{d}_{\mathrm{L}} \overline{\mathrm{s}}_{\mathrm{R}} \rightarrow \mathrm{s}_{\mathrm{L}} \overline{\mathrm{d}}_{\mathrm{R}}$. Thus, these may arise from the same LR interactions that are responsible for dynamical mass generation ${ }^{\star \star \star}$.

This approach contrasts with attempts to associate all weak isospin breaking with a single large ETC scale $[9,17]$. It is surprising how well one can do, but, as might be expected, to describe the origin of the top-bottom mass splitting in this way requires an inordinately fine adjustment of parameters. Were the current experimental limits on the deviation $\delta \rho$ of the $\rho$-parameter from 1 to be diminished to a few tenths of a percent, that scenario certainly would be incredible. In fact, it is much more natural to assume that there are many different ETC scales involved in generating quark and lepton masses and mixing angles, and we will explore that assumption in this paper.

One thing that is known is that simply scaling-up QCD fails to provide a large enough value for the strange quark mass, at least not if the requisite ETC scale $v_{\text {ETC }}^{(\mathrm{s})}$ is required to be more than $100 \mathrm{TeV}$ [18] or $200 \mathrm{TeV}$ [19] to avoid conflict with the limit on strangeness-changing neutral currents. However, assuming a

* Holdom [14] has emphasized the potential influence of color on the quark-lepton splitting of the second generation.

** For some numerical work in this direction, see Appelquist and Shapira [15].

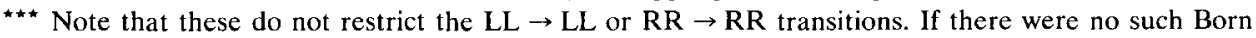
terms, the scale of ETC physics might be much lower. See, e.g. Nelson [16]. 
walking technicolor interaction up to the ETC scale, it would seem to be possible to realize quark masses on the order of hundreds of $\mathrm{MeV}$ for scales $v_{\mathrm{ETC}}^{\text {(s) }}$ as large as $1000 \mathrm{TeV}$ [7]. To account for a particle approximately 1000 times heavier than the strange quark, as the top quark must be, seems to be impossible from such large scales. Thus, one must require both condensate enhancement to account for the strange quark mass as well as introduce several ETC scales to account for the heavy quark and lepton masses. The specific picture we wish to entertain is this: we imagine that the technicolor interactions are walking or "slowly running", at least up to the lightest ETC scale, and that there may be several ETC scales. In this paper, we ignore most issues associated with mixing angles and $C P$-violation and focus on understanding how a very heavy top-quark and a very large top-bottom mass splitting might come about. However, certain multi-generational issues cannot be totally avoided because we must make some assumptions about the technifermion spectrum.

The spectrum of technifermions has been significantly constrained in several ways: The experimental limits on weak isospin breaking expressed by $\delta \rho$ limits the splittings among dynamical technifermion masses. This will be used elsewhere to constrain parameters in our effective lagrangian. More significantly, if there are many technifermions, they contribute to the variation in the isospin-symmetric contribution to the weak boson vacuum polarization tensor, which can be summarized in terms of a parameter called $S$ [20]. Existing data already provides significant restrictions, suggesting that there cannot be too many technifermion doublets and that the technicolor group is not too large [20,21]. Secondly, the greater the number of technifermions, the greater the ultimate number of global chiral symmetries and, consequently, the greater the number of pseudo-Goldstone bosons. One must require in the end that none be so light as to have been seen or to have influenced measureable parameters, such as $S$ [22]. This leads us to try to minimize the number of technifermions, in particular, to assume that there is a single generation of technifermions, [13]

$$
\left(\begin{array}{l}
N \\
E
\end{array}\right), \quad\left(\begin{array}{l}
U_{i} \\
D_{i}
\end{array}\right),
$$

where $i$ is a techniquark color index that will generally be suppressed.

If, as we assume, the technidown condensate $\langle\bar{D} D\rangle$ contributes both to the $\mathrm{b}, \mathrm{s}$, and $\mathrm{d}$ masses, then we are forced to look beyond the masses of the top and bottom quarks. The strange quark presents the greatest challenge because it must come from physics above $100 \mathrm{TeV}$ to satisfy the strangeness-changing neutral current constraints, and this determines a lower limit on the magnitude of the associated "technidown" condensate. To accomplish this necessitates that the technidown condensate $\langle\bar{D} D\rangle$ be much greater than is suggested by simply scaling-up QCD. This enhancement, we presume, arises from the persistence of technicolor forces 
and four-fermion interactions. As will be elaborated upon in sect. 2, the four-fermion interactions are necessarily large, although not so near critical as to require the sort of extreme fine-tuning encountered previously [9]. Allowing that the ETC scale associated with the top-quark mass could be relatively light, there is no real problem in a walking TC scenario generating a top-quark with a mass on the order of $100-200 \mathrm{GeV}$ [15]. Contrary to earlier attempts [9], in our scenario, the quark mass splittings are not driven by technifermion mass splittings, rather, the quark splittings are a direct consequence of the very different ETC scales posited, while the technifermion mass splittings are an indirect consequence that is necessary but unwanted. Nevertheless, we shall see that the technifermion contributions to $\delta \rho$ may be 10-100 times smaller than they were in those previous attempts [9]. However, as the ETC associated with the top quark becomes too light, certain other technifermion contributions to $\delta \rho$ become more difficult to restrain. Whether these myriad constraints can all be respected and still give a reasonable top and bottom quark is the subject of this paper.

\section{The general scenario and its constraints}

In this section, we shall begin to flesh out the picture described qualitatively in sect. 1 . We have indicated previously that we require the technicolor interactions to be asymptotically free, to generate confinement and chiral symmetry breaking, and slowly running, to justify the rather large ETC interactions from which they derive. This can be most easily accomplished by assuming that the non-abelian technicolor group is as small as possible, viz. $\mathrm{SU}_{2}^{\mathrm{TC}}$ and by admitting the minimum number of technifermions required to give the quarks and leptons mass. As given in eq. (1.2), for reasons that will become more apparent, we shall assume that our technifermions are technidoublets coming in 8 varieties that are carbon copies of a single generation of quarks and leptons. Thus, there are two "flavors" of techniquarks, each coming in 3 colors, and there are 2 flavors of technileptons. In other words, they are just like the ordinary quarks and leptons, except that they also carry technicolor. One possible difference, depending on whether neutrinos have mass, is that there certainly is a right-handed technineutrino.

It is perhaps worth noting that, for $\mathrm{SU}_{2}^{\mathrm{TC}}$, one can have at most 10 varieties of technifermions without changing the sign of the first term in the technicolor beta-function, thereby making the theory infrared rather than ultraviolet free, a completely different physical situation than the one we wish to consider. So, with this choice of technicolor group, we could not have more than one generation of technifermions. While one may entertain larger technicolor groups with more technifermions, a phenomenological reason for preferring a small group with as few technifermions as possible is the isospin-symmetric constraint $[20,21]$ men- 
tioned previously. This is the minimal technicolor model that is consistent with the usual $\mathrm{SU}_{3}^{\mathrm{C}} \otimes \mathrm{SU}_{2}^{\mathrm{L}} \otimes \mathrm{U}_{1}^{\mathrm{Y}}$ symmetries not being a part of the extended technicolor group.

The $\mathrm{SU}_{2}^{\mathrm{TC}}$ becomes strong on a scale of $\Lambda_{\mathrm{TC}}$ and causes chiral symmetry breaking through the formation of technifermion self-energies $\Sigma_{F}\left(p^{2}\right)$ associated with technifermion $F$. Being vector-like, the $\mathrm{SU}_{2}^{\mathrm{TC}}$ respects a global $\mathrm{SU}_{2}^{\mathrm{R}}$ so that, when the technifermions condense and break chiral symmetry, there remains a custodial weak isospin that ensures that $\rho=1$ in the limit that hypercharge and extended technicolor (ETC) interactions are neglected. The ETC interactions are primarily responsible for weak isospin breaking, but their influence is greatest at large scales, of order to the scale $v_{\text {ETC }}$ at which this symmetry is broken. The manner by which the constraints on the deviations of $\rho$ from 1 are satisfied is an extension of the idea behind walking technicolor, viz. the weak scale $F_{\pi}$ and, even more so, $\delta \rho$ are relatively insensitive to the high-momentum behavior of the fermion self-energies. In particular, so long as the splitting between members of an $\mathrm{SU}_{2}^{\mathrm{L}}$ doublet is sufficiently small in the infrared (by which we mean on the order of $\Lambda_{\mathrm{TC}}$ and below), it can be relatively large at high momenta without violating phenomenological constraints. The conditions by which differences among the $\Sigma_{F}\left(p^{2}\right)$ induce non-zero values of $\delta \rho \equiv \rho-1$ has been analyzed theoretically [23] and numerically [9] but, because this involves non-perturbative strong-interaction effects, it is admittedly difficult to make this quantitatively precise, as will be discussed further in sect. 4 below. However, it is important to estimate $\delta \rho$, for it is a constraint on new physics such as we are considering. The current experimental limits on $\delta \rho$ are about $1 \%$ [20], but future measurements promise to reduce this to a few tenths of a per cent, on the order of SM electroweak corrections themselves.

Now we must turn to the ETC interactions between the ordinary fermions and technifermions that induce the quark and lepton masses. Not having an understanding of their origin, we will be building up an effective field theory to the extent possible without specifying the precise nature of the ETC group and its breaking. This inductive rather than deductive approach is warranted by the lack of evidence as yet for technicolor generally, by our ignorance of the breaking pattern of non-vector-like theories, and most of all by the many failed attempts to guess an ETC group with a plausible symmetry breaking pattern that is realistic.

An immediate question is whether the effective four-fermion interactions must be restricted, for example, whether they are of the current-current type. Certainly we must expect vector bosons to mediate such exchanges, but, even though there are no fundamental scalar fields anticipated in this scenario, there may be pseudo-Goldstone bosons (PGB) or Higgs-like composite scalars arising from condensate fluctuations. It has been suggested [24] that a composite scalar below $M_{\text {ETC }}$ is an inevitable concomitant of condensate enhancement by four-fermi interactions, but whether it is narrow enough to be observable as a Higgs-like boson is another matter [25]. In any case, our model is like a standard model with 
four different Higgs doublets (one each for N, E, U, and D.) One can show that [9], if the four-fermion interactions are of current-current type, there is no mixing, i.e. each ordinary fermion gets mass from only one condensate. Thus, the mass of the top quark would come only from the condensate $\langle\bar{U} U\rangle$. Allowing scalar exchanges as well raises the possibility that $\langle\bar{D} D\rangle$ also contributes to the top mass. One might think this would lead to problems with the natural flavor-conservation in neutral currents, because it has been suggested that each fermion of definite charge should get mass from only one Higgs field [26]. However, the phenomenological argument that led to this suggestion applies only for light Higgs, whereas in this scenario, the Higgs is quite heavy, if it exists at all. So, although convenient to do so, we think it is unnecessary to restrict the form of the ETC interactions to be of current-current type.

In eq. (1.1), we indicated the general relation between the fermion mass and the condensate, but we did not define the condensate precisely. The technifermion condensate is commonly defined [7] as

$$
\langle\bar{F} F\rangle_{M f_{\mathrm{TC}}^{F}}=N_{\mathrm{TC}} \int^{M \mathbb{E T C}_{\mathrm{TC}}^{F}} \frac{\mathrm{d}^{4} k}{(2 \pi)^{4}} \frac{\Sigma_{F}\left(k^{2}\right)}{k^{2}+\Sigma_{F}\left(k^{2}\right)^{2}} .
$$

If $\Sigma(k)$ falls more slowly than $k^{-2}$ up to the ETC scale, as we are entertaining, then the condensate is indeed quite sensitive to the upper limit. However, to the extent that it begins to fall as $k^{-2}$ shortly beyond this $M_{\mathrm{ETC}}^{f F}$, it is really rather insensitive to physics much beyond this scale. For example, if the four-fermion interaction is mediated by exchange of a particle of mass $M_{\mathrm{ETC}}^{f F}$, the natural modification of the preceding expression would be

$$
\langle\bar{F} F\rangle_{M \mathrm{ETC}^{f f}}^{f}=\left(M_{\mathrm{ETC}}^{f F}\right)^{2} N_{\mathrm{TC}} \int \frac{\mathrm{d}^{4} k}{(2 \pi)^{4}} \frac{1}{k^{2}+\left(M_{\mathrm{ETC}}^{f F}\right)^{2}} \frac{\Sigma_{F}\left(k^{2}\right)}{\Sigma_{F}\left(k^{2}\right)^{2}+k^{2}} .
$$

Since there is no bare mass, $\Sigma(k)$ certainly vanishes asymptotically, so this integral converges. The effects of this sort of modification have been considered by Appelquist and Shapira [15]. To be more precise would require actually knowing the structure of the theory above $M_{\mathrm{ETC}}^{f F}$. The upshot is that the fermion mass should not be thought of as varying as $\left(M_{\text {ETC }}^{f F}\right)^{-2}$, since the self-energy does not necessarily fall as rapidly as $k^{-2}$, at least not until well above $M_{\text {ETC. In fact, at }}^{f F}$ least up to the scale $M_{\mathrm{ETC}}^{\mathrm{tU}}$, we suppose that as a result of walking and strong four-fermion interactions, the self-energy $\Sigma_{F}\left(k^{2}\right)$ falls very slowly [7] (roughly as $k^{-\gamma}$ with $\gamma<1$,) so that the condensate is a more-or-less sensitive function of the cutoff $M_{\mathrm{ETC}}^{f F}$. Immediately, many questions arise: Which technifermion condensates contribute to which fermion masses? What are the scales $M_{\text {ETC }}^{f F}$ associated with each fermion? How does explicit breaking of weak-isospin influence observables? 
In some ways, the quantity $m_{f}$ looks very much like a bare mass on scale $M_{\text {ETC }}^{f F}$, but since chiral symmetry breaking does not occur in the effective field theory at that scale, in fact, there is no bare mass. Another unintuitive feature is the nature of decoupling in such a picture. On the one hand, it is natural to associate the fermion mass $m_{f}$ with the scale $M_{\mathrm{ETC}}^{f F}$, but, on the other hand, the chiral symmetry breaking responsible for a non-zero $\Sigma_{F}$ is due to technicolor interactions that become strong at a much lower scale $\Lambda^{\mathrm{TC}}$. To resolve this paradox, it is helpful to observe that the momentum dependence of $\Sigma_{F}$ and the anomalous dimension of the operator $\bar{F} F$ at the scale $M_{\text {ETC }}^{f F}$ can in principle be determined from the physics on that scale and higher, but the normalization of $\Sigma_{F}$ and the existence of a non-zero $\langle\bar{F} F\rangle_{M{ }_{\mathrm{ETC}}^{F}}$ is determined by the technicolor dynamics characteristic of the lower scale $A^{\mathrm{TC}}$.

Although color must ultimately be included in the ETC group to allow for quark-lepton unification [5], the scale at which this occurs can be very high. Since the technicolor coupling is walking or slowly running at first, at the ETC scale responsible for the top mass, the Standard Model gauge interactions are not unified in the ETC group, since their gauge couplings are still too weak to be included. Therefore, at this scale, the massive ETC vector bosons will be singlets under $\mathrm{SU}_{3}^{\mathrm{QCD}} \otimes \mathrm{SU}_{2}^{\mathrm{L}} \otimes \mathrm{U}_{1}^{\mathrm{Y}}{ }^{\star}$. In that case, the exchange of ETC vector bosons can only connect technifermions and ordinary fermions having identical $\mathrm{SU}_{3}^{\mathrm{OCD}} \otimes \mathrm{SU}_{2}^{\mathrm{L}}$ $\otimes U_{1}^{Y}$ quantum numbers, so, for example, the $t$ is connected to the $U$ but not to the D. Actually, one can almost prove this "no mixing" result more generally [9], as follows: the current-current interaction contributing to technifermion condensates and ordinary fermion masses is of the $L_{\mu} R^{\mu}$ variety, where $L_{\mu}\left(R_{\mu}\right)$ is a current of left-handed (right-handed) fermions. Since the right-handed fermions are $\mathrm{SU}_{2}^{\mathrm{L}}$ singlets, then the $L_{\mu}$ current must also be an $\mathrm{SU}_{2}^{\mathrm{L}}$ singlet, so it cannot connect a $t$ to a $D$. However, this argument does not prohibit a technilepton condensate such as $\langle\bar{N} N\rangle$ from contributing to a quark mass, such as the $\mathrm{t}$, so the assumption that only a single condensate contributes to a given fermion mass (called monophagy [27]) is stronger than simply assuming current-current interactions. This monophagic structure is by no means generic to ETC models; there may be scalars, such as pseudo-Goldstone bosons, that not only may connect quarks to technileptons, but also may carry non-trivial $\mathrm{SU}_{3}^{\mathrm{QCD}} \otimes \mathrm{SU}_{2}^{\mathrm{L}} \otimes \mathrm{U}_{1}^{\mathrm{Y}}$ quantum numbers so that the $\langle\bar{D} D\rangle$ condensate could also contribute to the $t$ mass. Although the lore is that such a situation is to be avoided, it is not so clear in the present context. It has been pointed out that the masses of pseudo-Goldstone bosons can be lifted considerably in walking technicolor models [7], and relatively strong four-fermion interactions may increase them even more. We would expect the smallest PGB mass to be in the hundreds of $\mathrm{GeV}$, so it not obviously necessary to require

* But the breaking of the ETC group could also break another symmetry such as Pati-Salam. 
monophagy *. For simplicity we shall assume monophagy in our numerical work, but it may well be that this assumption is both inessential to our results and unnecessary in principle.

Up to what scale must the ETC group not include color? Even though the ETC coupling above $M_{\mathrm{ETC}}^{\mathrm{tU}}$ may be running, as its variation is only logarithmic, it will only be at much higher scales that it could become as small as the color coupling. We shall assume that this hypothesized pattern of monophagic four-fermion interactions persists at least up through the scale $M_{\mathrm{ETC}}^{\mathrm{sD}}$ responsible for the strange-quark mass. Then, at least in ladder approximation, there is no flavor mixing in the technifermion gap equation, except as the different flavors influence the running of the technicolor gauge coupling. Thus, the only nonzero $\lambda_{f F}$ in eq. (1.1) are those associated with the technifermion flavor $F$ having the same $\mathrm{SU}_{3}^{\mathrm{C}} \otimes \mathrm{SU}_{2}^{\mathrm{L}} \otimes \mathrm{U}_{1}^{\mathrm{Y}}$ quantum numbers as $f$. In this approximation, all quarks of charge $+2 / 3(-1 / 3)$ receive mass from the techni-U (techni-D) condensate. Similarly, all leptons of charge -1 receive mass from the techni-E, while the neutrinos, if their masses are non-zero, would have masses proportional to the techni-N.

This concludes our outline of the general scenario within which we seek a phenomenologically acceptable effective technicolor theory. If $M_{\text {ETC }}^{f F}$ is to have the significance of a vector boson mass, then $\Lambda_{f F}$ is proportional to $g_{\mathrm{ETC}}^{2}$, the square of an ETC gauge coupling, times some numerical factors of order 1 representing the coupling to the particular fermion $f$ and technifermion $F$. In the following, we shall simply take $\lambda_{f F} N_{\mathrm{TC}} / 4 \pi^{2}$ to be $\mathrm{O}(1)$, which may be a bit optimistic but well within the errors associated with other estimated quantities. Then the most stringent lower limit on the techni-D condensate $\langle\bar{D} D\rangle_{M_{\mathrm{ETC}}^{\mathrm{dD}}}$ is obtained from the lower limit on strangeness-changing neutral currents and the strange-quark mass. From eq. (1.1), we have $\langle\bar{D} D\rangle_{M_{\mathrm{ETC}}^{\mathrm{D}} \mathrm{D}}=m_{\mathrm{s}} v_{\mathrm{sD}}^{2}$. Now the lower limit, discussed previously, on $v_{\mathrm{sD}}$ of about $200 \mathrm{TeV}$ from $\mathrm{K}^{0}-\overline{\mathrm{K}^{0}}$ mixing together with $m_{\mathrm{s}} \approx 200$ $\mathrm{MeV}$ implies $\langle\bar{D} D\rangle_{M_{\mathrm{ETC}}^{\mathrm{dP}}} \geqslant(2 \mathrm{TeV})^{3}$. Assuming this lower limit on the $\langle\bar{D} D\rangle$ condensate is realized, then to account for $m_{\mathrm{b}}$ requires that $v_{\mathrm{bD}} \approx 40 \mathrm{TeV}$. The observed value of $\mathrm{B}^{0}-\overline{\mathrm{B}^{0}}$ mixing suggests that $v_{\mathrm{bD}} \geqslant 20 \mathrm{TeV}$, even if the associated mixing angle is as large as the Cabibbo angle. So mixing is consistent with our inferred lower limit of about $40 \mathrm{TeV}$. The upper limit on $\mathrm{D}^{0}-\overline{\mathrm{D}^{0}}$ mixing suggests that $v_{\mathrm{cU}} \geqslant 35 \mathrm{TeV}$, again assuming mixing on the order of the Cabibbo angle. Together with the observed valued of $m_{\mathrm{c}}$, this implies the lower limit $\langle\bar{U} U\rangle_{M_{\mathrm{ETC}}^{\mathrm{cU}}} \geqslant$ $(1.2 \mathrm{TeV})^{3}$. If we assume that $m_{\mathrm{t}}$ is on the order of $100 \mathrm{GeV}$, then this lower on $\langle\bar{U} U\rangle$ suggests that $v_{\mathrm{ETC}}^{\mathrm{tU}}$ on greater than about $4 \mathrm{TeV}$. It is difficult to be more precise, but these various constraints will be seen to be satisfied by the parameters of our model.

* By way of contrast, this property was essential to the approach in ref. [9]. 


\section{Structure of the ETC group}

Given this general scenario and the preceding constraints, we will now further develop our model for the heaviest quarks of all, the $b$ and $t$, and show that their splitting can be explained in a phenomenologically acceptable way. What we need to do is to develop a scheme for estimating the differences in self-energies $\Sigma_{U}$ and $\Sigma_{\mathrm{D}}$ associated with the techni-U and techni-D, respectively, and correspondingly, the differences in the condensates $\langle\bar{U} U\rangle_{M_{\mathrm{ETC}}^{\mathrm{LU}}}$ and $\langle\bar{D} D\rangle_{M_{\mathrm{ETC}}^{\mathrm{bD}}}$. At the same time, we need to estimate how large a contribution to the $\rho$-parameter is implied by these differences. We also need to know the contributions of the top and bottom quarks to the $\rho$-parameter, but as these masses are hard up to $M_{\mathrm{ETC}}^{\mathrm{tU}}$ and $M_{\mathrm{ETC}}^{\mathrm{bD}}$, respectively, the result will be very nearly the classic result of the SM [28,29], and we will not bother about them further. To estimate how the weak-isospin splitting affects the $\rho$-parameter, we will study the gap equation in ladder approximation as in previous work $[8,9]$. This is admittedly a crude estimate, but to do better requires a reliable method of treating the strongly-interacting technicolor forces.

While we do not wish to specify precisely what the ETC group is above $M_{\mathrm{ETC}}^{\mathrm{tU}}$, we cannot avoid discussing it altogether if we wish to discuss the bottom-quark mass and the nature of the gap equation in the range $M_{\mathrm{ETC}}^{\mathrm{tU}}<p<M_{\mathrm{ETC}}^{\mathrm{bD}}$. Above $M_{\mathrm{ETC}}^{\mathrm{tU}}$, the top and techni-U fields become united in an irreducible representation of the effective ETC symmetry group. For example, if they transformed under an $\mathrm{SU}_{3}^{\mathrm{A}}$, then they might by unified in ETC triplets,

$$
\left(\begin{array}{c}
U_{1} \\
U_{2} \\
t
\end{array}\right)_{\mathrm{L}}\left(\begin{array}{c}
U_{1} \\
U_{2} \\
t
\end{array}\right)_{\mathrm{R}}\left(\begin{array}{c}
D_{1} \\
D_{2} \\
b
\end{array}\right)_{\mathrm{L}} .
$$

Note that both the left- and right-handed $U$ and $t$ fields must be so unified, so that one will get four-fermion interactions of the form $\overline{t_{\mathrm{R}}} t_{\mathrm{L}} \overline{U_{\mathrm{L}}} U_{\mathrm{R}}$ or, possibly, $\overline{t_{\mathrm{R}}} t_{\mathrm{L}} \overline{U_{\mathrm{R}}} U_{\mathrm{L}}$, giving rise to mass terms for the top from the techni-U condensate. Inasmuch as the left-handed particles are members of weak doublets, then the $b_{\mathrm{L}}$ and $D_{\mathrm{L}}$ fields must be unified in precisely the same fashion as the $t_{\mathrm{L}}$ and $U_{\mathrm{t}}$. An obvious way to have the $\mathrm{b}$ quark avoid getting a mass at this ETC scale is to have the $b_{\mathrm{R}}$ be a singlet under the generators that unify the $t$ and $U$. For example, the ETC group could be $\mathrm{SU}_{3}^{\mathrm{A}} \otimes \mathrm{SU}_{2}^{\mathrm{B}}$ at this stage, where $D_{\mathrm{R}}$ is a singlet under $\mathrm{SU}_{3}^{\mathrm{A}}$ and a doublet under $\mathrm{SU}_{2}^{\mathrm{B}}$ and $b_{\mathrm{R}}$ a singlet under both. This generic form $\mathrm{G}^{\mathrm{A}} \otimes \mathrm{G}^{\mathrm{B}}$ of the ETC interactions above $M_{\mathrm{ETC}}^{\mathrm{tU}}$ is the one we shall adopt [30,31], but first we shall consider and dismiss an alternative scheme.

A less obvious possibility for preventing the $b$ from deriving a mass from the $M_{\text {ETC }}^{\text {tU }}$ scale exploits the fact that the technicolor group might be real, such as the $\mathrm{SU}_{2}^{\mathrm{TC}}$ that we have chosen *. The three multiplets displayed in (3.1) transform as

* This mechanism was illustrated for leptons to avoid a neutrino mass by Sikivie et al. [32]. 
triplets as before, but the $b_{\mathrm{R}}$ is combined not with the technidoublet $D_{\mathrm{R}}$ but with the conjugate doublet $\widetilde{D_{\mathrm{R}}}$ in a $3^{*}$ of $\mathrm{SU}_{3}^{\mathrm{ETC}}$,

$$
\left(\begin{array}{c}
D_{2} \\
-D_{1} \\
b
\end{array}\right)_{\mathrm{R}}
$$

Since $\overline{D_{\mathrm{L}}} D_{\mathrm{R}}$ condenses in an $\mathrm{SU}_{2}^{\mathrm{TC}}$ singlet, it makes no contribution to the $\mathrm{b}$ mass. Unfortunately, this mechanism ensures that the $b$ remains massless, that is, embedding this $\mathrm{SU}_{3}^{\mathrm{ETC}}$ in a larger group will not allow the $\mathrm{b}$ to acquire a mass through four-fermi interactions of the form $\bar{R} L \bar{L} R$. This can be seen from the fact that, with this assignment, the b mass arises from a symmetric product of $\bar{R} L$ whereas the condensate occurs only in the anti-symmetric product $\bar{L} R$. Therefore they cannot be combined to form an $\mathrm{SU}_{3}^{\mathrm{ETC}}$ invariant four-fermion interaction *.

Therefore, the only viable sort of model is the previous type in which the effective ETC group above $M_{\text {ETC }}^{\mathrm{tU}}$ is not simple but of the form $\mathrm{G}^{\mathrm{A}} \otimes \mathrm{G}^{\mathrm{B}}$. In a scheme of this kind, what sort of four-fermion interactions are to be expected? Because $t$ and $U$ are unified to give the top mass term, we must expect terms of the form $\overline{t_{\mathrm{L}}} t_{\mathrm{R}} \overline{t_{\mathrm{R}}} t_{\mathrm{L}}$ and $\overline{U_{\mathrm{L}}} U_{\mathrm{R}} \overline{U_{\mathrm{R}}} U_{\mathrm{L}}$, with coefficients proportional to $\left(1 / M_{\mathrm{ETC}}^{\mathrm{tU}}\right)^{2}$ and, as we shall discuss below, these enter the gap equation for the techni-U self-energy and top mass. Because left-handed fields are doublets, there are also $\overline{D_{\mathrm{L}}} U_{\mathrm{R}} \overline{U_{\mathrm{R}}} D_{\mathrm{L}}$ but, because we assume that the condensates preserve charge conservation, these will not contribute to the gap equation.

There will also be terms of the form $\overline{D_{\mathrm{L}}} D_{\mathrm{R}} \overline{D_{\mathrm{R}}} D_{\mathrm{L}}$ associated with the scale $M_{\mathrm{ETC}}^{\mathrm{tU}}$. This can be seen quite generally as follows: above the scale $M_{\mathrm{ETC}}^{\mathrm{tU}}$, to the extent that $\mathrm{G}^{\mathrm{A}} \otimes \mathrm{G}^{\mathrm{B}}$ is a good symmetry, there are no ETC interactions between $D_{\mathrm{L}}$ and $D_{\mathrm{R}}$ as these fields to do not couple to a common generator. Below $M_{\mathrm{ETC}}^{\mathrm{tU}}$ the remaining unbroken symmetry is the vectorlike technicolor interaction, which is a linear combination of generators of $G^{A}$ and $G^{B}$. The massive gauge bosons must then also be linear combinations of generators of these two symmetries. This suggests that the four-fermion interaction among the $\mathrm{D}$ may be negative, reflecting that it is the residuum of contributions that must cancel the technicolor interaction. This would tend to depress rather than enhance the down condensate $\langle\bar{D} D\rangle$ and, if true, would make it hard to account for the strange-quark mass. Since we do not understand the ETC dynamics and there may be several different effects contributing to these four-fermion interactions, we have preferred to leave the sign and magnitude of the four-fermion interactions as free parameters.

Presumably, then, there is some higher scale $M_{\mathrm{ETC}}^{\mathrm{bD}}$ responsible for the b quark mass. What symmetry group emerges above that scale? There would appear to be

* The possibility exists of a mass arising from higher-dimensional interactions, such as a six-fermion operator, but this will not be pursued here. 
two possibilities: in the first, the two sets of generators of $G^{A}$ and $G^{B}$ may intertwine, for example, the ETC group could be $\mathrm{SU}_{6}$. In this case, the b mass arises simply from the four-fermion operators analogous to the $t$ mass, but with $v_{\mathrm{ETC}}^{\mathrm{tU}}$ replaced by $v_{\mathrm{ETC}}^{\mathrm{dD}}$. Alternatively, the direct product structure might be retained, for example, $\mathrm{SU}_{3}^{\mathrm{A}} \otimes \mathrm{SU}_{2}^{\mathrm{B}}$ may become $\mathrm{SU}_{3}^{\mathrm{A}} \otimes \mathrm{SU}_{3}^{\mathrm{B}}$. At first sight, it would appear that this would be incapable of generating a $b$ mass, because the generators connecting the $b_{\mathrm{R}}$ to $D_{\mathrm{R}}$ commute with those connecting the $b_{\mathrm{L}}$ to the $D_{\mathrm{L}}$. However, in such a scheme, the massive vector bosons coming from the breaking of each $\mathrm{SU}_{3}$ can mix proportional to the scale at which the symmetry breaks down to technicolor [30,31], but this depends on a better understanding of the origin of the ETC symmetry breaking scales. To describe the possibilities, this has been modeled by Higgs fields. It may be that there are intermediate scales, and the pattern might be more complicated than we have described, for example

$$
\mathrm{SU}_{3}^{\mathrm{A}} \otimes \mathrm{SU}_{3}^{\mathrm{B}} \stackrel{\nu_{\mathrm{ETC}}^{\mathrm{tD}}}{\longrightarrow} \mathrm{SU}_{3}^{\mathrm{A}} \otimes \mathrm{SU}_{2}^{\mathrm{B}} \stackrel{\nu_{\mathrm{ETC}}^{\mathrm{tU}}}{\longrightarrow} \mathrm{SU}_{2}^{\mathrm{A}} \otimes \mathrm{SU}_{2}^{\mathrm{B}} \stackrel{\nu_{1}}{\longrightarrow} \mathrm{SU}_{2}^{\mathrm{TC}}
$$

However, because technicolor would switch off in the techni-D gap equation at the intermediate scale above which $\mathrm{SU}_{2}^{\mathrm{A}} \otimes \mathrm{SU}_{2}^{\mathrm{B}}$ emerges, and it is the technidown condensate that must be enhanced to account for the strange-quark mass, we will simply assume that the scale $v_{\mathrm{I}}$ is approximately the same as $v_{\text {ETC }}^{\text {tU }}$.

\section{Quantitative estimates using the gap equation}

We wish to quantify the preceding remarks to determine the viability of this scenario. Unfortunately, chiral symmetry breaking is a non-perturbative phenomenon for which there is at present no reliable calculational scheme. A certain amount of insight has been gained from hadron dynamics that presumably result from QCD. In order to quantify our conclusions, following closely earlier work [8,9], we will develop a model for inferring the magnitude of the technifermion self-energy and condensate. As previously discussed, the quark and lepton masses are proportional to the technifermion condensates (eq. (1.1)) which, in turn, are functionals of the technifermion self-energy. What we shall do is to estimate how the technifermion self-energies are determined by the parameters of a particular technicolor scenario (beta function, four-fermion coupling, ETC mass scales, etc.) Therefrom, we can estimate the corresponding condensate and thereby infer the associated quark and lepton masses. While we would be the first to admit that, at present, our approximations are rather crude, we have no other choice. Originally [19], the condensate was estimated simply by "scaling up" the quark condensate in QCD. However, we wish to consider more general gauge theories having different dynamical properties than QCD, and simply "scaling up" will not accurately describe this physics. 


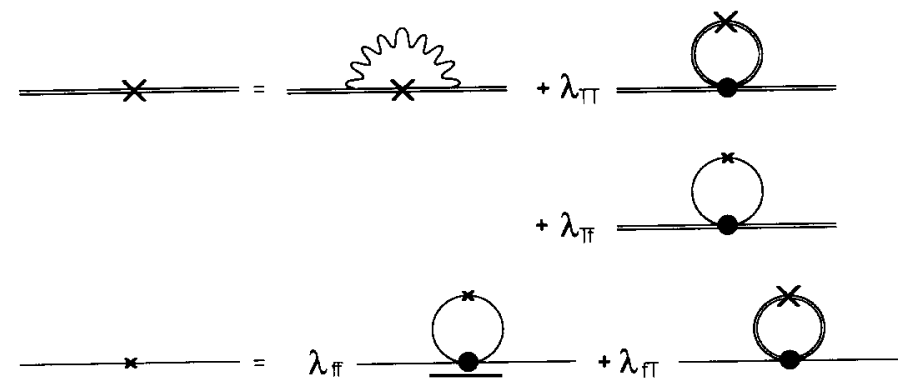

Fig. 2. The Dyson-Schwinger gap equation for the techni-up and the top quark. The techni-up propagator is represented by a double line; its self energy by a large cross. The top quark is represented by a single line; its self energy, by a small cross. The technigluon is a wavy line; the ETC four-fermion coupling represented by a dot.

The method we shall use to calculate the fermion self-energy is to find solutions of the Dyson-Schwinger gap equation [33,34] (fig. 2). This equation is a non-perturbative integral equation for the fermion propagator. The exact equation requires knowledge of the gauge-boson propagator and gauge-boson - fermion vertex to all orders in the coupling constant, which in principle also depend on the fermion self-energies. A common approximation [35] is to make a kind of "ladder" approximation in which vertex corrections and vacuum polarization is approximated by replacing the gauge coupling constant by the running coupling [36] * However, it is clearly inadequate to use a perturbative approximation in the "infrared" regime where the technicolor coupling becomes large. We cannot allow it to blow up as it would in perturbation theory, so we arbitrarily cut off the running coupling when it is equal to three times $\alpha_{c}$, the minimum value that would break chiral symmetry in the quenched approximation ${ }^{\star}$. Finally, we perform calculations in Landau gauge so that wave function renormalization is absent. The gap equation is solved numerically for the fermion self-energy or, more precisely, it is solved twice, once for the techni-U, and once for the techni-D ${ }^{\star \star \star}$. This is necessary in order to infer the effects of weak-isospin breaking on the condensate and on the $\rho$-parameter.

The equation for the self-energy of the techni-U is slightly more straightforward, so we will begin with a discussion of it. As previously mentioned, we are considering an $\mathrm{SU}_{2}$ technicolor gauge force with ETC interactions represented by four-fermion interactions. Since the effective field theory is only valid up to $M_{\mathrm{ETC}}^{\mathrm{tU}}$, we cut off virtual momenta at that scale. However, this should not effect our solution, because details of the high-energy regime can be adsorbed by changes in the four-fermion coupling. Above this scale, the top quark will become unified

* For a discussion on the effects of including higher-order terms in the kernel, see ref. [36].

** Our numerical work shows that once the coupling is bigger than the critical value, the solution does not depend on where we truncate it.

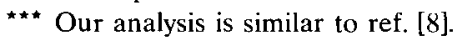


with the techni-U. One complication is that the top-quark mass is not so much smaller than we imagine the techni-U mass to be. Therefore, we must allow the top self-energy to feed back into the techni-U self-energy, leading to the following coupled set of gap equations, depicted in fig. 2 :

$$
\begin{aligned}
\Sigma_{\mathrm{U}}(p)= & \int_{0}^{M_{\mathrm{ETC} 2}^{\mathrm{UU}}} \mathrm{d} k^{2} \frac{\alpha\left(\max \left(p^{2}, k^{2}\right)\right)}{4 \alpha_{\mathrm{c}}} \frac{k^{2}}{\left(\max \left(p^{2}, k^{2}\right)\right)} \frac{\Sigma_{\mathrm{U}}(k)}{k^{2}+\Sigma_{\mathrm{U}}^{2}(k)} \\
& +\lambda_{\mathrm{TT}} \int_{0}^{M_{\mathrm{ETT}}^{\mathrm{tUT}}} \frac{\mathrm{d} k^{2}}{4 \pi^{2}} \frac{k^{2}}{M_{\mathrm{ETC}}^{\mathrm{tU} 2}} \frac{\Sigma_{\mathrm{U}}(k)}{k^{2}+\Sigma_{\mathrm{U}}^{2}(k)} \\
& +\lambda_{\mathrm{Tt}} \int_{0}^{M_{\mathrm{ETC}}^{\mathrm{UUT} 2}} \frac{a k^{2}}{4 \pi^{2}} \frac{k^{2}}{M_{\mathrm{ETC}}^{\mathrm{tU} 2}} \frac{\Sigma_{\mathrm{t}}(k)}{k^{2}+\Sigma_{\mathrm{t}}^{2}(k)} \\
\Sigma_{\mathrm{t}}(p)= & \lambda_{\mathrm{tt}} \int_{0}^{M_{\mathrm{ETC}}^{\mathrm{tU} 2}} \frac{\mathrm{d} k^{2}}{4 \pi^{2}} \frac{k^{2}}{M_{\mathrm{ETC}}^{\mathrm{tU} 2}} \frac{\Sigma_{\mathrm{t}}(k)}{k^{2}+\Sigma_{1}^{2}(k)} \\
& +\lambda_{\mathrm{tT}} N_{\mathrm{TC}} \int_{0}^{M_{\mathrm{ETC}}^{\mathrm{tU} 2}} \frac{\mathrm{d} k^{2}}{4 \pi^{2}} \frac{k^{2}}{M_{\mathrm{ETC}}^{\mathrm{tU} 2}} \frac{\Sigma_{\mathrm{U}}(k)}{k^{2}+\Sigma_{\mathrm{U}}^{2}(k)}
\end{aligned}
$$

where $\lambda_{\mathrm{TT}}, \lambda_{\mathrm{Tt}}, \lambda_{\mathrm{tT}}$, and $\lambda_{\mathrm{tt}}$ are the various four-fermion coupling strengths, and $N_{\text {TC }}$ is the dimension of the techni-U representation under technicolor. In our model, $N_{\text {TC }}=2$. The technicolor interaction strength is given by the running coupling $\alpha(p)$, this will appear in combination with $\alpha_{\mathrm{c}}$, so we define [8]

$$
a(p) \equiv \frac{\alpha(p)}{4 \alpha_{\mathrm{c}}}= \begin{cases}a_{\Lambda_{\mathrm{TC}}} /\left(1+\frac{2 a_{A_{\mathrm{TC}}}}{A} \ln \left(\frac{p^{2}}{\Lambda_{\mathrm{TC}}^{2}}\right)\right) & p>\Lambda_{\mathrm{TC}} \\ a_{\Lambda_{\mathrm{TC}}} & p \leqslant \Lambda_{\mathrm{TC}}\end{cases}
$$

where $\alpha_{\mathrm{c}}$ is the critical coupling in the quenched approximation $\left(\alpha_{\mathrm{c}}=\pi / 3 C_{2}(R)\right)$, and $a_{A_{\mathrm{TC}}}=3 / 4$, corresponding to $\alpha=3 \alpha_{\mathrm{c}}$, the value at which we have assumed the coupling strength saturates. The parameter $A$ is defined by $A \equiv 1 / b \alpha_{c}$, where $b$ is the coefficient of the $\alpha^{2}$ term in the beta function. For an $\mathrm{SU}_{N}$ gauge theory with $n_{\mathrm{f}}$ Dirac fermions,

$$
A=\frac{3\left(N^{2}-1\right)}{N\left(\frac{11}{3} N-\frac{2}{3} n_{\mathrm{f}}\right)} .
$$


We note that, in this local approximation, the top self-energy $\Sigma_{t}(p)$ actually has no momentum dependence and may be called $m_{\mathrm{t}}$. This allows one to simplify the coupled equations and to bring them to the form

$$
\begin{aligned}
\Sigma_{\mathrm{U}}(p)= & \int_{0}^{M_{\mathrm{E} T \mathrm{C}}^{\mathrm{U} 2} \mathrm{~d} k^{2}} \frac{\alpha\left(\max \left(p^{2}, k^{2}\right)\right)}{4 \alpha_{\mathrm{c}}} \frac{k^{2}}{\left(\max \left(p^{2}, k^{2}\right)\right)} \frac{\Sigma_{\mathrm{U}}(k)}{k^{2}+\Sigma_{\mathrm{U}}^{2}(k)} \\
& +\lambda_{U} \int_{0}^{M_{\mathrm{ETC}}^{\mathrm{UU} 2}} \mathrm{~d} k^{2} \frac{k^{2}}{M_{\mathrm{ETC}}^{\mathrm{tU} 2}} \frac{\Sigma_{\mathrm{U}}(k)}{k^{2}+\Sigma_{\mathrm{U}}^{2}(k)}, \\
m_{\mathrm{t}}= & \lambda_{\mathrm{t}} \int_{0}^{M_{\mathrm{ETC}}^{\mathrm{tU} 2}} \mathrm{~d} k^{2} \frac{k^{2}}{M_{\mathrm{ETC}}^{\mathrm{tU} 2}} \frac{\Sigma_{\mathrm{U}}(k)}{k^{2}+\Sigma_{\mathrm{U}}^{2}(k)},
\end{aligned}
$$

where

$$
\lambda_{\mathrm{U}} \approx \frac{1}{4 \pi^{2}} \lambda_{\mathrm{TT}}+\frac{N_{\mathrm{TC}}}{4 \pi^{2}} \lambda_{\mathrm{Tt}}\left(\frac{\lambda_{\mathrm{tI}} / 4 \pi^{2}}{1-\lambda_{\mathrm{tt}} / 4 \pi^{2}}\right)
$$

and

$$
\lambda_{\mathrm{t}} \approx \frac{\underline{N}_{\mathrm{IC}} \lambda_{\mathrm{LI}}}{4 \pi^{2}}\left(\frac{1}{1-\lambda_{\mathrm{tt}} / 4 \pi^{2}}\right) .
$$

A value for $\lambda_{\mathrm{U}}$ may be estimated by noting that (neglecting possible group-theory factors) $\lambda_{\mathrm{TT}} / 4 \pi^{2} \approx\left(g^{2}\left(M_{\mathrm{ETC}}^{\mathrm{tU}}\right)\right) /\left(4 \pi^{2}\right) \approx 0.7$, and $g^{2}\left(M_{\mathrm{ETC}}^{\mathrm{tU}}\right)$ falling as $\mathrm{SU}_{2}$ with 8 flavors. From this it is not unreasonable to assume that both $\lambda_{\mathrm{t}}$ and $\lambda_{\mathrm{U}}$ are order 1.

Before describing the techni-D, we shall discuss some general features of eq. (4.5). There are two parameters that we may adjust which qualitatively change the solution. One is the rate at which the technicolor coupling runs, here the parameter $A$. The other is the strength of the four-fermion interaction, here given by $\lambda_{\mathrm{U}}$. What we shall attempt to do by adjusting these parameters is to increase the value of the techniquark condensate (and hence the quark and lepton masses), without affecting the value of $F_{\pi}$. As we shall later show, $F_{\pi}$ is determined largely by the infrared behavior of $\Sigma(p)$, whereas the condensate is much more sensitive to the high-momentum behavior.

At first we shall set $\lambda_{\mathrm{U}}=0$, and vary $A$. In fig. 3 we graph $\Sigma(p) / F_{\pi}$ for several different values of $A$. With increasing $A$, we are exploring technicolor theories with smaller and smaller beta functions. These are known as "walking" technicolor models [9]. When $A$ is large the self-energy $\Sigma(p)$ falls much more slowly with momentum, and there is enhancement of quark and lepton masses over a similar theory with smaller $A$. 
We now set $A=2.25$ (the value for an $\mathrm{SU}_{2}$ technicolor with 8 Dirac fermions) and vary $\lambda_{\mathrm{U}}$. The results are shown in fig. 4. As in the previous case, we have increased the value of $\Sigma(p)$ at high momentum without significantly changing it at low momenta.

In the models we shall consider, we will chose a value for $A$, and obtain an acceptable quark-mass spectrum by adjusting the four-fermion coupling strengths and the ETC scale at which the quarks couple to the techniquarks. We will also show that this is possible without "fine-tuning" these parameters, as previously indicated.

The gap equation for the techni-D is slightly more complicated. In analogy to the earlier case, we integrate up to the ETC scale of the bottom quark ( $\left.M_{\mathrm{ETC}}^{\mathrm{bD}}\right)$. However, as has been previously discussed, the technicolor interaction no longer affects the techni-D above the ETC scale of the top quark (the color force does though and, despite its size, its effects are not entirely negligible). In order to model this, we simply "turn off" the technicolor force above this scale (see fig. 5). We also need to include a four-fermion interaction at this scale. Hence, for the techni-D we have an $\mathrm{SU}_{2}$ technicolor that runs up to a scale $M_{\mathrm{ETC}}^{\mathrm{tU}}$ and then shuts off. We have two four-fermion terms, one at $M_{\mathrm{ETC}}^{\mathrm{tU}}$, and one at $M_{\mathrm{ETC}}^{\mathrm{bD}}$. The gap equation for the techni- $\mathrm{D}$ is (in final form)

$$
\begin{aligned}
\Sigma_{\mathrm{D}}(p)= & \int_{0}^{M_{\mathrm{ETC}}^{\mathrm{bD} 2}} \mathrm{~d} k^{2} \frac{\alpha\left(\max \left(p^{2}, k^{2}\right)\right)}{4 \alpha_{\mathrm{c}}} \frac{k^{2}}{\left(\max \left(p^{2}, k^{2}\right)\right)} \frac{\Sigma_{\mathrm{D}}(k)}{k^{2}+\Sigma_{\mathrm{D}}^{2}(k)} \\
& +\lambda_{\mathrm{D}} \int_{0}^{M_{\mathrm{ETC}}^{\mathrm{bD} 2}} \mathrm{~d} k^{2} \frac{k^{2}}{M_{\mathrm{ETC}}^{\mathrm{bD} 2}} \frac{\Sigma_{\mathrm{D}}(k)}{k^{2}+\Sigma_{\mathrm{D}}^{2}(k)} \\
& +\lambda_{\mathrm{Dt}} \theta\left(M_{\mathrm{ETC}}^{\mathrm{tU}}-p\right) \int_{0}^{M_{\mathrm{ETC}}^{\mathrm{UU} 2}} \mathrm{~d} k^{2} \frac{k^{2}}{M_{\mathrm{ETC}}^{\mathrm{tU} 2}} \frac{\Sigma_{\mathrm{D}}(k)}{k^{2} \mp \Sigma_{\mathrm{D}}^{2}(k)}
\end{aligned}
$$

and the bottom mass is

$$
m_{\mathrm{b}}=\lambda_{\mathrm{b}} \int_{0}^{M_{\mathrm{ETC}}^{\mathrm{bD} 2}} \mathrm{~d} k^{2} \frac{k^{2}}{M_{\mathrm{ETC}}^{\mathrm{bD} 2}} \frac{\Sigma_{\mathrm{D}}(k)}{k^{2}+\Sigma_{\mathrm{D}}^{2}(k)},
$$

where $\lambda_{\mathrm{Dt}}$ is the four-fermion interaction at the scale of the top quark, and all other four-fermion terms are defined in a manner similar to eq. (4.7). This is identical to eq. (4.5) except that the running coupling has no technicolor contribution above $M_{\mathrm{ETC}}^{\mathrm{tU}}$, and there is an additional four-fermion interaction below $M_{\mathrm{ETC}}^{\mathrm{tU}}$. (Note also that the techni-U integral is cut off at $M_{\mathrm{ETC}}^{\mathrm{tU}}$.)

In models in which the ETC group above $M_{\text {ETC }}^{\text {bD }}$ remains of the product-group type [30,31], such as $\mathrm{SU}_{3}^{\mathrm{A}} \otimes \mathrm{SU}_{3}^{\mathrm{B}}$, there are some minor modifications to be made 


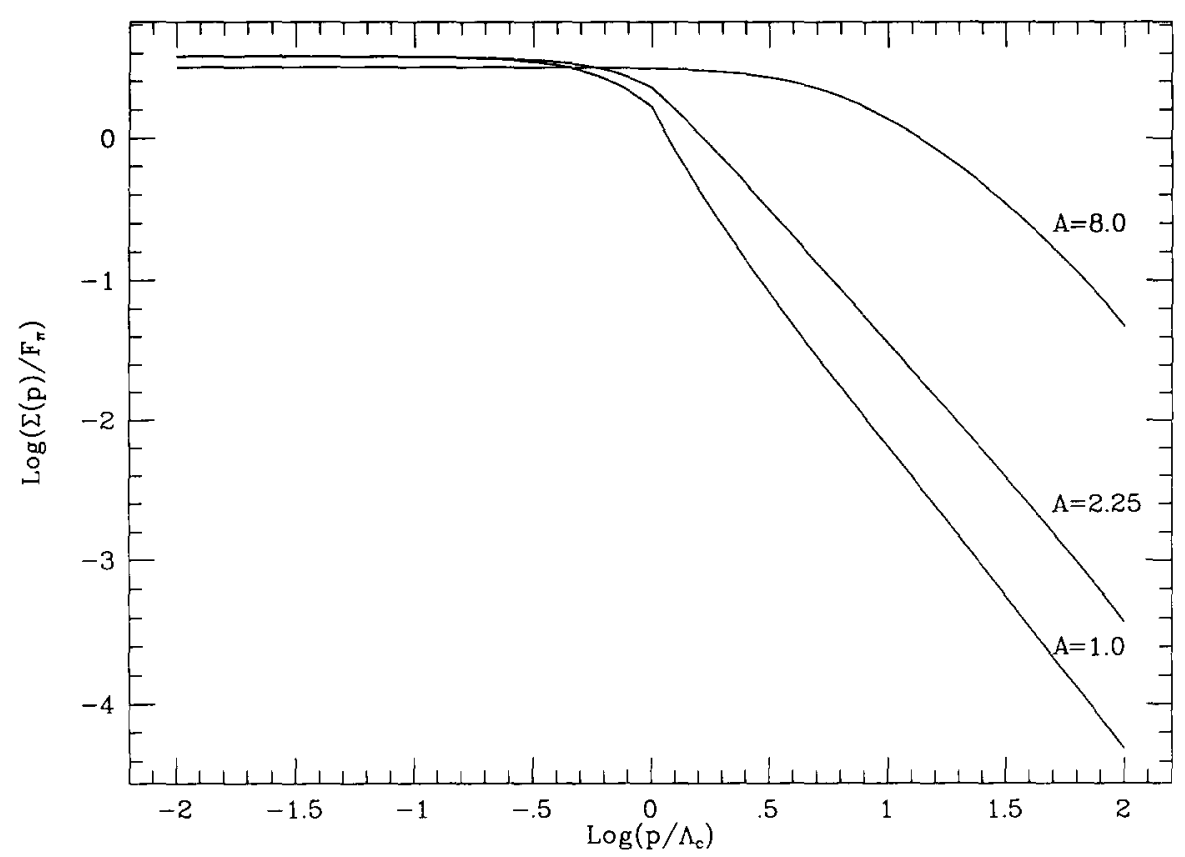

Fig. 3. $\Sigma(p) / F_{\pi}$ for several different values of the slope parameter $A$, with no four-fermion enhancement $\left(\lambda_{U}=0\right)$.

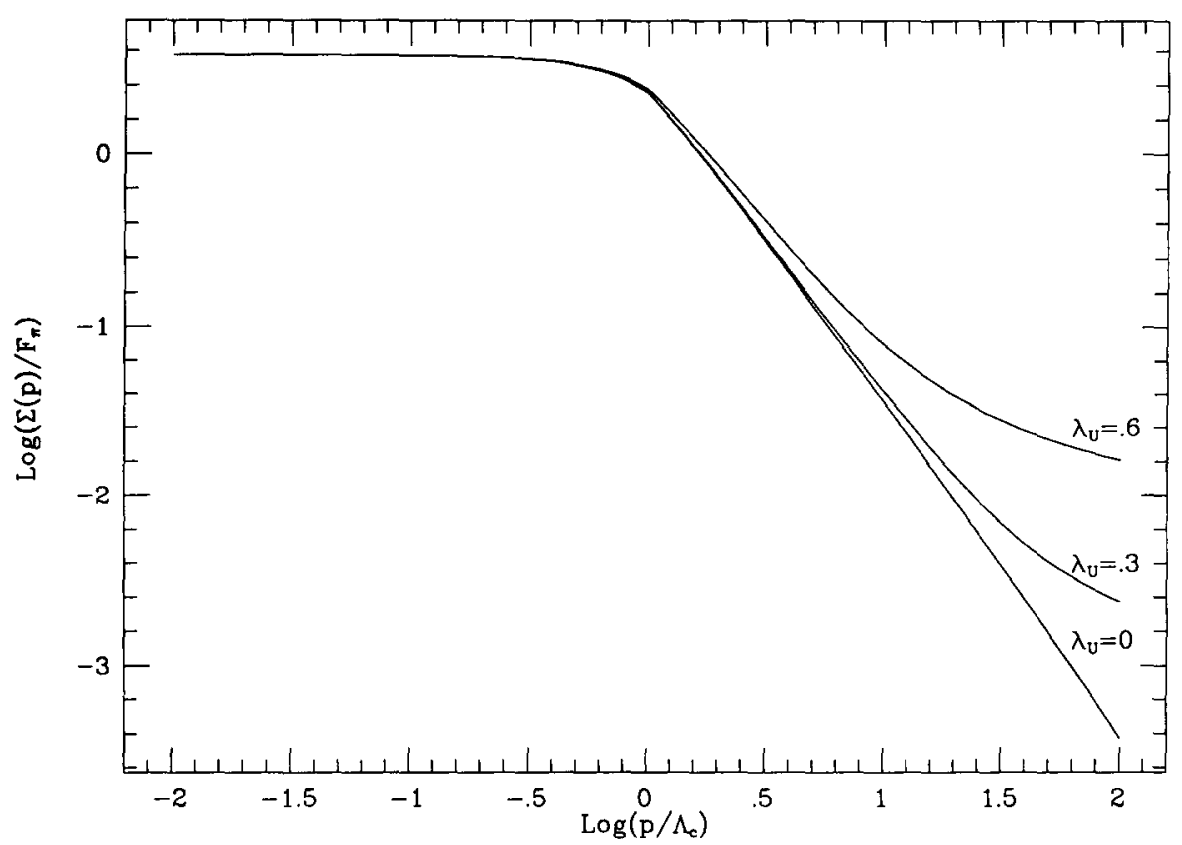

Fig. 4. $\Sigma(p) / F_{\pi}$ for several different values of the parameter $\lambda_{\mathrm{U}}$, with $A=2.25$ (the value for an SU(2) technicolor with 8 Dirac fermions). 


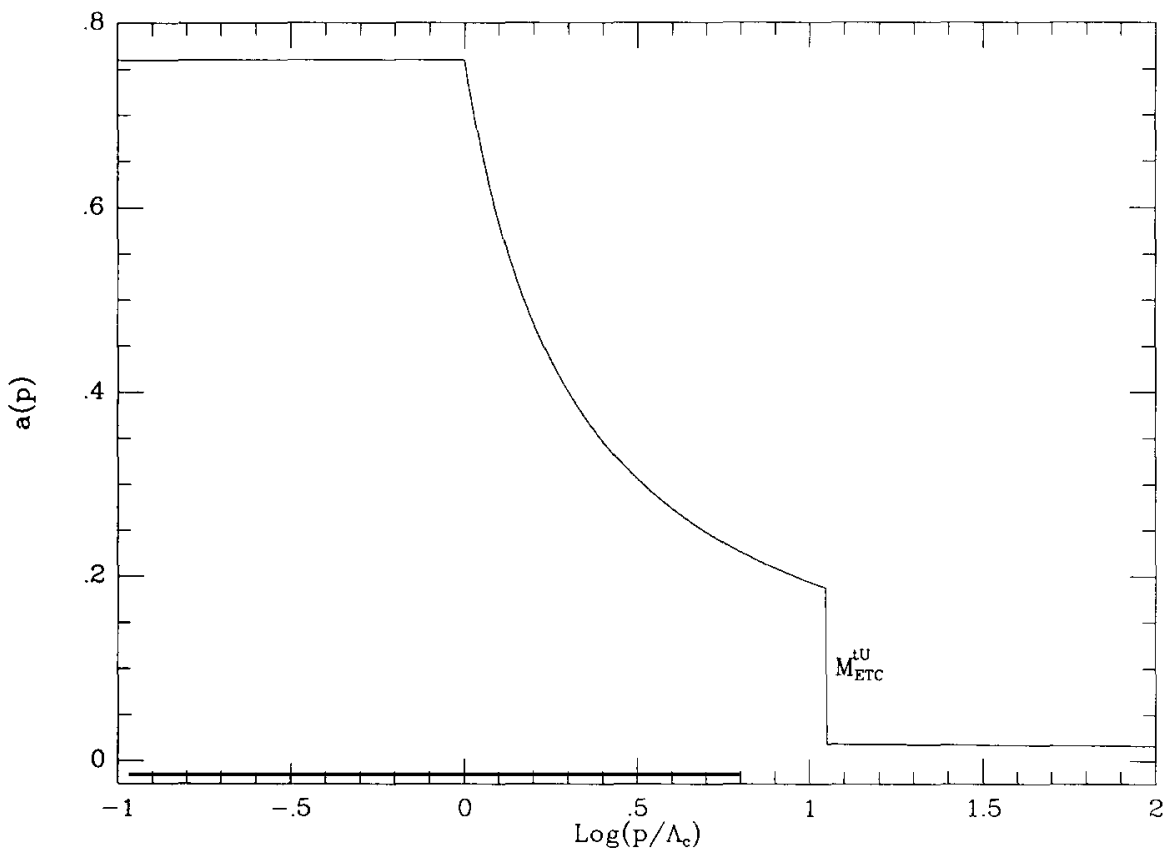

Fig. 5. The running coupling $a(p)=\alpha(p) / 4 \alpha_{c}$.

in eqs. (4.9) and (4.10). The integral in eq. (4.10) and in the second term of eq. (4.9) would then be cut off at $M_{\mathrm{ETC}}^{\mathrm{tU}}$ since, above that scale, the vector boson mixing responsible for the coupling between $D_{\mathrm{L}}$ and $D_{\mathrm{R}}$ switches off. While qualitatively very important, this would alter our quantitative conclusions only slightly, leading to a somewhat smaller $v_{\text {ETC }}^{\text {bD }}$ scale to account for $m_{\mathrm{b}}$ and slightly different values for $\lambda_{\mathrm{D}}$.

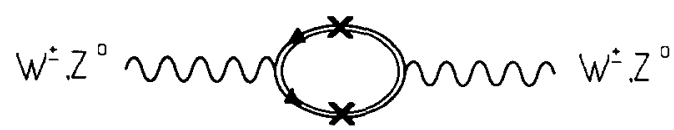

(a)

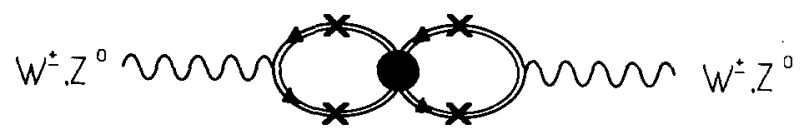

(b)

Fig. 6. (a) The Pagels-Stokar approximation for calculating $F_{\pi}$. The double lines are technifermions, and the crosses are mass insertions. (b) "Direct" contributions to $\delta \rho$. The four-fermion operators are represented by a dot. 
Once we have calculated the technifermion self-energies we must use them to construct a physical quantity so that our dimensionful units will have meaning. The quantity we shall consider is the technipion decay constant $F_{\pi}=250 \mathrm{GeV}$. From this we shall also obtain a set of limits on $\delta \rho$. In order to calculate $F_{\pi}$, we use a slightly modified Pagels-Stokar approximation [23], as shown in fig. 6(a)

$$
\begin{aligned}
& F_{ \pm}^{2}=\frac{N}{1 \pi^{2}} \int \mathrm{d} k^{2}\left[\frac{k^{2}\left\{\Sigma_{\mathrm{U}}^{2}\left(k^{2}\right)+\Sigma_{\mathrm{D}}^{2}\left(k^{2}\right)\right\}+\Sigma_{\mathrm{U}}^{2}\left(k^{2}\right) \Sigma_{\mathrm{D}}^{2}\left(k^{2}\right)}{\left\{k^{2}+\Sigma_{\mathrm{U}}^{2}\left(k^{2}\right)\right\}\left\{k^{2}+\Sigma_{\mathrm{D}}^{2}\left(k^{2}\right)\right\}}\right], \\
& F_{0}^{2}=\frac{N}{16 \pi^{2}} \int \mathrm{d} k^{2}\left[\frac{k^{2} \Sigma_{\mathrm{U}}^{2}\left(k^{2}\right)+\frac{1}{2} \Sigma_{\mathrm{U}}^{4}\left(k^{2}\right)}{\left\{k^{2}+\Sigma_{\mathrm{U}}^{2}\left(k^{2}\right)\right\}^{2}}+\frac{k^{2} \Sigma_{\mathrm{D}}^{2}\left(k^{2}\right)+\frac{1}{2} \Sigma_{\mathrm{D}}^{4}\left(k^{2}\right)}{\left\{k^{2}+\Sigma_{\mathrm{D}}^{2}\left(k^{2}\right)\right\}^{2}}\right],
\end{aligned}
$$

where $N$ is the number of technifermion doublets; in our model $N=4 \times 2=8^{*}$. The basic structure of technicolor theories gives

$$
M_{\mathrm{W}}^{2}=\frac{g_{2}^{2} F_{ \pm}^{2}}{4}, \quad M_{\mathrm{Z}}^{2}=\frac{\left(g_{1}^{2}+g_{2}^{2}\right) F_{0}^{2}}{4},
$$

and

$$
\rho=\frac{M_{\mathrm{W}}^{2}}{M_{\mathrm{Z}}^{2} \cos ^{2} \theta_{\mathrm{W}}}=\frac{F_{ \pm}^{2}}{F_{0}^{2}},
$$

hence

$$
\delta \rho=\rho-1=\frac{F_{ \pm}^{2}-F_{0}^{2}}{\underline{F_{0}^{2}}} .
$$

When $\Sigma_{\mathrm{U}}=\Sigma_{\mathrm{D}}$ we have $F_{ \pm}=F_{0}$ and hence $\delta \rho=0$. We call contributions to $\delta \rho$ that results from $\Sigma_{U} \neq \Sigma_{D}$ "indirect" contributions. Our numerical results will focus on these, but we shall also discuss certain "direct" contributions below.

$F_{ \pm}$and $F_{0}$ set the scale of the technifermion self-energies, from which we may then calculate fermion masses and require agreement with experiment. A set of parameters that lead to a phenomenologically acceptable spectrum of quark masses are presented in table 1. These correspond to a $200 \mathrm{MeV}$ strange-quark mass, a $5 \mathrm{GeV}$ bottom-quark mass, and a $100 \mathrm{GeV}$ top-quark mass. In addition, our assumption of strong ETC interactions, $\lambda_{\mathrm{T}} \approx 1$, implies $g_{\text {ETC }} \approx 2 \pi / \sqrt{N_{\mathrm{TC}}}=4.4$ in the calculation of $v^{q^{T}}$. There are several points worthy of note about this data. Consider the first entry. It has no four-fermion enhancement, but is the result of a "slowly running" technicolor $(A=2.25)$. While it does provide an appropriate quark-mass spectrum, it does not meet the strangeness-changing neutral current

* Strictly speaking, we should sum over each technifermion-fermion multiplet, but in the approximation in which color and hypercharge interactions are neglected, each doublet contributes equally. 


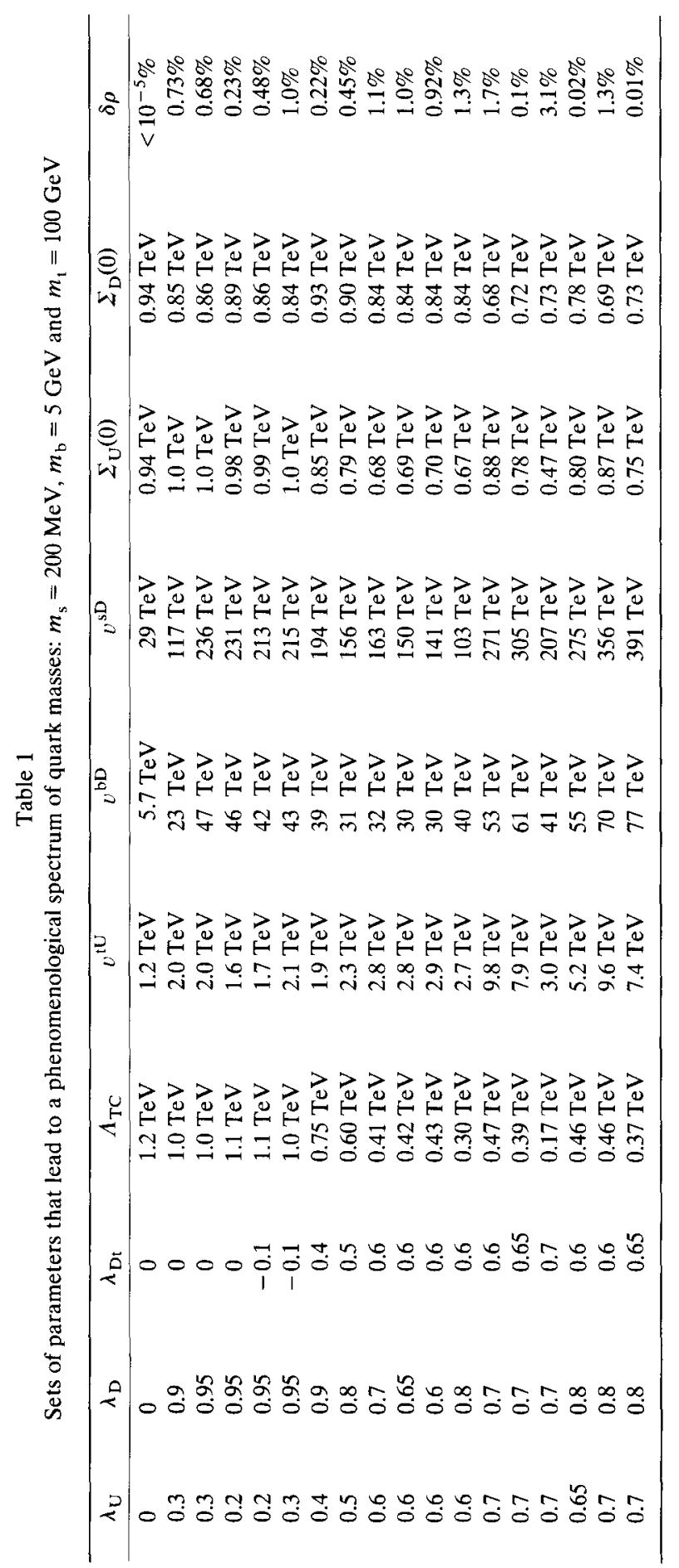


constraint that $v^{\mathrm{sD}}>100-200 \mathrm{TeV}$. In subsequent entries, we show that, with substantial four-fermion enhancement, this constraint can be satisfied in a variety of ways. The easiest method involves four-fermion couplings at both the top and bottom scales in the techni-D sector (in addition to the top scale in the techni-U sector). To illustrate the interplay of the four-fermion forces, the first few entries of table 1 indicate that without enhancement at the top scale, a larger four-fermion coupling at the bottom scale may be needed in the techni-D. However, if enhancement occurs at both scales, the four-fermion couplings need not be as large. On the other hand, if $\lambda_{\mathrm{Dt}}$ is negative, then it may be seen that $\lambda_{\mathrm{D}}$ must be very near critical in order to provide the necessary enhancement of $\langle\bar{D} D\rangle$.

For sufficiently large values of the four-fermion couplings, $\Sigma(p)$ becomes essentially momentum independent, as in the original Nambu-Jona-Lasinio model

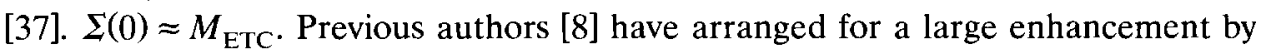
adjusting their four-fermion couplings extremely close to this critical value. Typically, adjustments of 1 part in $10^{4}$ were required. In addition to this "fine-tuning", these scenarios would have an unacceptably large $\delta \rho$ if the top-bottom mass splitting resulted from different four-fermion couplings only. As table 1 demonstrates, the present multi-scale scenario avoids these difficulties. In the first place, our ETC scales are low enough to give acceptable quark masses, and yet high enough to avoid giving observable flavor-changing neutral current effects. Secondly since our mass splittings are primarily the result of different ETC scales, we have much less problem keeping $\delta \rho$ small. Viewing the last three columns of table 1 , one sees that $\delta \rho \neq 0$ is really a function of $\Sigma_{U}(0) \neq \Sigma_{D}(0)$. In fact, by a judicious choice of four-fermion couplings, it is possible to make $\delta \rho$ arbitrarily small.

We note that another possible source of contributions to $\delta \rho$ is what we shall call "direct" contributions [38], as shown in fig. 6(b). These contributions are generated by four-fermion interactions of the form $\left(\mathrm{g}^{2} / M_{\mathrm{ETC}}^{2}\right) \overline{T_{R}} T_{\mathrm{R}} \overline{T_{R}} T_{\mathrm{R}}$. They exist independently of whether or not $\Sigma_{U}=\Sigma_{D}$ as they result from direct isospin breaking in the ETC sector. In order to estimate the size of these contributions, we assume $\Sigma_{\mathrm{U}}=\Sigma_{\mathrm{D}}$ and $\Sigma(p)=\Sigma(0)(\Sigma(0) / p)^{2 \gamma}$, where $\gamma \approx 0.5$ for a "walking" theory. If our momentum integrals run from $\Sigma(0)$ to $M_{\mathrm{ETC}}^{\mathrm{tU}}$, we find from fig. 6(b)

$$
\begin{aligned}
\delta \rho_{\text {direct }} & \approx \frac{\Sigma^{2}(0)}{F_{\pi}^{2}}\left(\frac{1}{2 \gamma}\right)^{2} \frac{\Sigma^{2}(0)}{M_{\text {ETC }}^{\text {tU } 2}} \frac{g_{\text {ETC }}^{2} N_{\text {doublet }}^{2}}{\left(8 \pi^{2}\right)^{2}}\left[1-\left(\frac{\Sigma^{2}(0)}{M_{\text {ETC }}^{\text {tU } 2}}\right)^{2 \gamma}\right]^{2} \\
& \approx \frac{\Sigma^{2}(0)}{16 F_{\pi}^{2}} \frac{N_{\text {doublet }}^{2}}{\pi^{2}} \frac{g_{\text {ETC }}^{2}}{4 \pi^{2}} \frac{\Sigma^{2}(0)}{M_{\text {ETC }}^{\mathrm{tU} 2}} \\
& \approx \frac{\Sigma^{2}(0)}{4 \pi^{2} v^{\mathrm{tU} 2}} \\
& \leq 1 \% .
\end{aligned}
$$


For many of the parameters in the table, $\delta \rho_{\text {direct }}$ is on the order of a few tenths of one percent. While this is acceptable, it could be problematic if $M_{\mathrm{ETC}}^{\mathrm{tU}}$ were any smaller. On the other hand, since $\delta \rho_{\text {direct }}$ is inversely proportional to $\left(M_{\mathrm{ETC}}^{\mathrm{tU}}\right)^{2}$, as this scale becomes larger, this contribution to $\delta \rho_{\text {direct }}$ rapidly becomes negligible. Thus, while we cannot be very precise, we would not be at all surprised if the next generation of precision measurements determine that $\delta \rho$ does not agree with the predictions based solely on SM physics.

An analysis similar to the one we have performed for $\delta \rho$ should be done for the isospin symmetric parameter $S$. A rough estimate [20,21] gives

$$
S=2.1+0.4\left(N_{\mathrm{TC}}-4\right)
$$

for a model with 4 technidoublets. In our scenario $N_{\mathrm{TC}}=2$ and hence $S \approx 1$. This value is within the current experimental limits. We note that this estimate for $S$ is based upon the assumption that technicolor is simply "scaled up" QCD. We have previously argued that in our scenario technicolor would have very different properties than QCD, and hence this calculation may be suspect. More work is needed to find out how phenomena such as "walking" and four-Fermi enhancement will affect this parameter.

\section{Conclusions}

In this paper, we have shown that, from a phenomenological point of view, a technicolor scenario can accommodate a mass as large as a top quark in the range of $100-200 \mathrm{GeV}$ and a mass splitting as large as $m_{\mathrm{t}}-m_{\mathrm{b}}$. The ingredients include persistent technicolor interactions, slowly running if not walking, together with strong ETC forces in the form of four-fermion interactions. It is actually more difficult to account for $m_{\mathrm{s}}$ than $m_{\mathrm{t}}$ given that the scale $v^{\mathrm{sD}} \geq 100 \mathrm{TeV}$. In fact, if this scale were an order of magnitude larger, as is sometimes required, it would require delicate fine-tuning of the four-fermion interaction $\lambda_{\mathrm{SD}}$ to be very near its critical value.

We have shown that, with strong ETC interactions, one can account for the strange and top quark masses. Unlike previous treatments $[9,39]$ it is not necessary to fine-tune these interaction strengths. To this end, we will expand on the meaning of fine-tuning and compare it with the notion of naturalness. Naturalness [40] is the idea that a parameter can be understood to be naturally small if, when it is set zero, a higher symmetry emerges that guarantees it remains zero, even after radiative corrections. In this sense, bare parameters are not adjusted to compensate radiative corrections to high precision, but this notion is in fact more general than naturalness. The concept of "no fine-tuning" is that, to account for observa- 
tions, the tree-level lagrangian parameters on some scale should not have to be adjusted to an accuracy greater than the characteristic size of radiative corrections due to relevant, i.e. renormalizable, interactions. Thus, if to account for the top mass, one must choose a four-fermi interaction to be within one-part in a million of a certain critical value when the characteristic size of radiative corrections to that parameter due to other interactions, such as color, are $1 \%$, then we would call that fine-tuning. Looking back at our table, we see that the allowed variation in our four-fermion interaction couplings is much greater than the characteristic size of the radiative corrections to that coupling that can be anticipated due to the technicolor, color, etc. In that sense, their values may be fortuitous and it is a challenge to explain their origin, but they cannot be said to be finely-tuned.

In fact, the magnitude of $C P$ violation associated with the imaginary part of the kaon mass matrix is sometimes used to set a lower limit on the order of $1000 \mathrm{TeV}$ or more, at least in models in which the imaginary part is associated with a scale in the same manner we have assumed for the real parts. This large scale would seem to be impossible for us to accommodate without the sort of fine-tuning of four-fermion couplings that we are trying to avoid. So we must assume that, whatever the eventual explanation of $C P$ violation, it will provide for a natural suppression of its strength. The only viable mechanism for accounting for $C P$ violation in technicolor models of electroweak symmetry breaking seems to be spontaneous breakdown of $C P$ invariance [41], but there are a number of allowed alternatives even in this context, and we must assume that one is chosen that either suppresses the imaginary part relative to the real part or simply suppresses the strength of strangeness-changing neutral currents altogether. As discussed previously, the limits on other flavor-changing neutral currents provide much weaker constraints on the scale of new physics.

We have not discussed the spectrum and interactions of pseudo-Goldstone bosons. In scenarios with walking technicolor or strong four-fermion interactions, their masses typically lie in the hundreds of $\mathrm{GeV}$, avoiding one of the primary phenomenological difficulties with older technicolor models. The masses of pseudo-Goldstone bosons will be further enhanced in the present context. The discussion of their properties is a subject for future work, but it apparently has the consequence that monophagy may not be a necessary requirement to bring models such as ours into conformity with the limits on flavor-changing neutral currents.

We have ignored any question of anomalies associated with the ETC groups, because we have not specified the mechanism responsible for the various ETC scales. In the spirit of these models, it is natural to assume there are other fermions that condense as a result of other gauge forces, and that these condensates are responsible for the ETC scales. The representation content of these additional fermions must satisfy the anomaly constraints. Simple examples of such models can be found in ref. [32]. It is challenge to construct such anomaly-free, plausible ETC models. 
Finally, we reiterate a word of caution. All of our numerical results stem from what are essentially uncontrolled approximations of non-perturbative phenomena. While there is some evidence (largely from QCD) that these approximations are qualitatively accurate we have no quantitative handle on the size of our errors. Until such time as reliable non-perturbative methods exist, we must simply express the hope that our results should at least provide order-of-magnitude estimates for these phenomena. But this is all we want at this point - to decide whether or not TC and ETC models for fermion masses have a chance of being viable. Our conclusion is that, if TC interactions are persistent and ETC interactions are multi-scale and strong, then it seems possible to account for the quark and lepton mass spectrum. It seems clear that, allowing for arbitrary scales, we could accommodate three generations and known mixing angles. But, given that the spectrum can be so generated, the challenge for future work is to find a realistic ETC group that can plausibly account for the various scales and couplings.

We would like to thank T.A. Appelquist, T. Takeuchi and R. Wijewardhana for discussions about this work. Portions of this research were accomplished while the authors were participants in the Workshop on Physics Far Below the Planck Energy at the Institute for Theoretical Physics at the University of California, Santa Barbara. We would like to thank the Workshop coordinators, especially, T. Zee, for facilitating our participation. Portions of this work were supported by the Department of Energy and by the National Science Foundation (Grant PHY8904035.)

\section{References}

[1] CDF Collaboration, K. Sliwa et al.) FERMILAB-CONF-90-93-E, May 1990

[2] F. Dydak, XXIV Int. Conf. on High energy physics, Singapore, August 1990

[3] S. Weinberg, Phys. Rev. D13 (1976) 974, D19 (1979) 1277;

L. Susskind, Phys. Rev. D20 (1979) 2619;

E. Farhi and L. Susskind, Phys. Rep. 74 (1981) 277

[4] S. Dimopoulos and L. Susskind, Nucl. Phys. B155 (1979) B155

[5] E. Eichten and K. Lane, Phys. Lett. B90 (1980) 125

[6] B. Holdom, Phys. Rev. D24 (1981) 1441; Phys. Lett. B198 (1987) 535; Phys. Rev. Lett. 60 (1988) 1233

[7] T. Appelquist, D. Karabali and L.C.R. Wijewardhana, Phys. Rev. Lett. 57 (1986) 957;

T. Appelquist and L.C.R. Wijewardhana, Phys. Rev. D35 (1987) 774; D36 (1987) 568

[8] T. Appelquist, M. Einhorn, T. Takeuchi and L.C.R. Wijewardhana, Phys. Lett. B220 (1989) 223;

T. Takeuchi, Phys. Rev. D40 (1989) 2697

[9] T. Appelquist, M. Einhorn, T. Takeuchi, L.C.R. Wijewardhana, Phys. Lett B232 (1989) 211

[10] S. Kaptanoglu and N.K. Pak, Fortschr. Phys. 30 (1982) 451;

R. Kaul, Rev. Mod. Phys. 55 (1983) 449

[11] C. Vafa and E. Witten, Nucl. Phys. B234 (1984) 173

[12] S. Raby, S. Dimopoulos and L. Susskind, Nucl. Phys. B169 (1980) 373

[13] S. Dimopoulos, Nucl. Phys. B168 (1980) 69 
[14] B. Holdom, Phys. Rev. Lett. 60 (1988) 1233

[15] T. Appelquist and O. Shapira, Phys. Lett. B249 (1990) 83

[16] A. Nelson, Phys. Rev. D38 (1988) 2875

[17] S.F. King and D.A. Ross, Phys. Lett. B228 (1989) 363; Southampton preprint SHEP-88/89-17 (April, 1990)

[18] P. Langacker, In Proc. 1990 DPF Summer Study on High energy physics research directions for the decade, Snowmass, Colorado, June 25, 1990, to be published

[19] S. Dimopoulos and J. Ellis, Nucl. Phys. B182 (1981) 505

[20] M. Peskin and T. Takeuchi, Phys. Rev. Lett. 65 (1990) 964

[21] W.J. Marciano and J.L. Rosner, Phys. Rev. Lett. 65 (1990) 2963

[22] M. Golden and L. Randall, Fermilab preprint 90/83-T (1990)

[23] T. Appelquist, M. Einhorn, T. Takeuchi and L.C.R. Wijewardhana, Phys. Rev. D41 (1990) 3192

[24] R.S. Chivukula, A. Cohen and K. Lane, Nucl. Phys. B343 (1990) 554

[25] T. Appelquist, J. Terning and L.C.R. Wijewardhana, Phys. Rev. D44 (1991) 871.

[26] S. Glashow and S. Weinberg, Phys. Rev. D15 (1977) 1958

[27] J. Ellis, M. Gaillard, D. Nanopoulos and P. Sikivie, Nucl. Phys. B182 (1981) 529

[28] M. Veltman, Acta Phys. Pol. B8 (1977) 475

[29] M. Chanowitz, M. Furman and I. Hinchliffe, Phys. Lett. B78 (1978) 285

[30] A.J. Buras, S. Dawson and A.N. Schellekens, Phys. Rev. D27 (1983) 1171

[31] S.F. King, Phys. Lett. B229 (1989) 253

[32] P. Sikivie et al. Nucl. Phys. B173 (1980) 189

[33] F.J. Dyson, Phys. Rev. 75 (1949) 1736; J. Schwinger, Proc. Nat. Acad. Sci. 37 (1951) 452

[34] T. Maskawa and H. Nakajima, Prog. Theor. Phys. 52 (1974) 13; 54 (1976) 860; R. Fukuda and T. Kugo, Nucl. Phys. B117 (1976) 250

[35] K. Higashijima, Phys. Rev. D29 (1984) 1228; P. Castoria and S.Y. Pi, Phys. Rev. D31 (1985) 411; R. Casalbuoni, S. De Curtis, D. Dominici and R. Gatto, Phys. Rev. Lett. B150 (1985) 295

[36] T. Appelquist, K. Lane and U. Mahanta, Phys. Rev. Lett. 61 (1988) 1553; U. Mahanta, Phys. Rev. Lett. 62 (1989) 2349; Phys. Lett. B225 (1989) 181

[37] Y. Nambu and G. Jona-Lasinio, Phys. Rev. 122 (1961) 345; 124 (1961) 246

[38] T. Appelquist, M. Bowick, E. Cohler and A. Hauser, Phys. Rev. Lett. 53 (1984) 1523; Phys. Rev. D31 (1985) 1676

[39] R.R. Mendel and V.A. Miransky, University of Western Ontario preprint UWO-DAM-15/91 (1991)

[40] G. ' $t$ Hooft, in Recent developments in gauge theories, ed. G. 't Hooft et al. (Plenum Press, New York, 1980)

[41] E. Eichten, K. Lane and J. Preskill, Phys. Rev. Lett. 45 (1980) 225; reviewed by K. Lane, Physica Scripta 23 (1981) 1005 